\title{
Exosomes Secreted by Epidural Adipose-Derived Stem Cells Promote Malignancy and EMT in B Cell Non-Hodgkin Lymphoma by Increasing S100A4 Expression
}

\section{Yan Wang}

affiliated hospital of qingdao university

Zhu Guo

affiliated hospital of qingdao university

\section{Guoqing Zhang}

affiliated hospital of qingdao university

\section{Baoxin Shang}

affiliated hospital of qingdao university

\section{Xiaolin Wu}

affiliated hospital of qingdao university

Chensheng Qiu

affiliated hospital of qingdao university

\section{Hongfei Xiang}

affiliated hospital of qingdao university

Bohua Chen ( $\nabla$ bhchen@hotmail.com )

affiliated hospital of qiingdao university

\section{Research}

Keywords: Exosomes Secreted, Epidural Adipose, Lymphoma

Posted Date: January 8th, 2021

DOI: https://doi.org/10.21203/rs.3.rs-141262/v1

License: (c) (i) This work is licensed under a Creative Commons Attribution 4.0 International License. Read Full License 


\section{Abstract}

Our previous study revealed that epidural adipose-derived stem cells (ADSCs) respond to lung cancer cells and are involved in forming premetastatic niches. The biological functions of epidural ADSCs in lymphoma are still unclear. In this study, we first confirmed the B cell lymphoma-promoting effects of epidural ADSC exosomes and then compared differentially expressed genes in lymphoma cells before and after exosome treatment. In ADSC exosome-treated lymphoma cells, S100A4 expression was much higher than that in cells cultured alone. S100A4 levels had a direct relationship with EMT in cells and were also associated with the survival of lymphoma patients. Then, we tested the roles of ADSC exosomes and S100A4 in the lymphoma-promoting impacts of ADSCs. Our results showed that ADSC exosomes could foster the proliferation, EMT and invasion of lymphoma cells, as could the increase in S100A4 expression. S100A4 inhibition in two lymphoma cell lines suppressed the lymphoma cell proliferation's ADSC exosome-mediated promotion, invasion and EMT in vitro. Moreover, S100A4 inhibition attenuated metastasis and tumour growth, and ADSC exosomes promoted tumour progression, as demonstrated in a nude mouse model of lymphoma. On the basis of these data, ADSC exosomes foster B cell lymphoma progression by increasing S100A4 expression in lymphoma cells.

\section{Introduction}

Metastatic epidural spinal cord compression (MESCC) is a devastating complication of malignancy, which occurs in approximately $5 \%$ of patients with cancer ${ }^{1}$. Breast, prostate, and lung cancer each account for $15 \%$ to $20 \%$ of all cases of MESCC; non-Hodgkin lymphoma, myeloma, and renal cell carcinoma each account for $5 \%$ to $10 \%^{2}$. MESCC may arise from hematogenous metastasis to vertebral body initial, and then invasion of the epidural space in most tumors, or direct through the intervertebral foramina to spread into the epidural space, such as lymphoma and Pancoast tumors of the lung s.4 $^{3,4}$ Metastases involving the dural sac, root sleeves, and their contents are major causes of neurological dysfunction ${ }^{5}$.

Spinal lymphoma is more frequently caused by secondary dissemination from advanced disease, and which is more often associated with non-Hodgkin lymphoma, especially the diffuse large B-cell subtype ${ }^{5}$. Different from other neoplastic diseases of the spine, the epidural soft tissue mass of spinal lymphoma is more obvious, and reveals circumferential compression of the spinal cord that epidural soft tissue mass of tumor grows around the dura and spreads into the intervertebral foramen ${ }^{3-5}$.

Epidural fat is the last barrier in the progression of MESCC, and in which adipose-derived mesenchymal stem cells (ADSCs) has been proved to be activated and exhibit cancer promoting properties in MESCC from lung cancer ${ }^{6}$. The special invasion mode in spinal lymphoma demonstrate that spinal lymphoma may have a closer relationship with epidural fat or ADSCs than other spinal metastases.

Our previous work revealed that epidural adipose-derived stem cells (ADSCs) were involved in the formation of premetastatic niches in the epidural fat and responded to lung cancer cells to promote 
tumour metastasis and proliferation ${ }^{6}$. However, the role of epidural ADSCs in non-Hodgkin lymphoma spinal metastasis and the exact mechanisms underlying their effects remain poorly understood. Exosomes are extracellular vesicles that transmit lipids, messenger RNA, DNA, microRNA and proteins and function as crucial players in intercellular communication ${ }^{7}$. Exosomes derived from stromal cells are integral in the formation of the tumour microenvironment and involved in all stages of cancer progression ${ }^{8}$. Understanding the effects of epidural ADSC exosomes in the spinal microenvironment may allow ADSCs to serve as preventative targets for cancer therapy.

The small protein S100A4 is a member of the $\mathrm{S} 100$ family of $\mathrm{Ca}^{2+}$-binding proteins ${ }^{9}$. Moreover, it takes charge of diverse intracellular pathways and by regulating the intracellular $\mathrm{Ca}^{2+}$ concentration, it causes different effects ${ }^{10}$. S100A4 is a famous makers who characterize epithelial-mesenchymal transition $(E M T)^{11}$. EMT is a composite biological process. In this process endothelial cells make use of a mesenchymal phenotype, together with the typical cell functions and morphology which contains the acquisition of contractile properties and cellular motility ${ }^{12}$. In some cancer cells, S100A4 takes the responsibility of the ability to form metastases, and promote cell invasiveness and motility ${ }^{13}$. However, at present, we know nothing about S100A4's role in the lymphoma spinal metastasis-promoting influence of ADSCs.

In our research, we isolated exosomes from epidural ADSCs and assessed the influence of ADSC exosomes on B cell non-Hodgkin lymphoma cells. Significant promotion of proliferation and invasion were observed in two lymphoma cell lines, and transcriptomic analyses were performed to understand genes with differential expression after ADSC exosome treatment. Significantly increased S100A4 expression was found in lymphoma cells treated with ADSC exosomes, and gene set enrichment analysis (GSEA) revealed an enrichment in EMT-associated pathways. We predicted that ADSC exosomes could increase S100A4 expression in lymphoma cells, which supported lymphoma cell invasion and proliferation. In the research, we examined the potential roles of S100A4 in the communication between ADSCs and lymphoma cells through exosomes.

\section{Materials And Methods}

\section{Cell culture}

As previously described, from healthy adult donors who underwent posterior lumbar discectomy, human epidural ADSCs were obtained so as to treat or lumbar disc herniation or lumbar fracture ${ }^{6}$. According to the ethical guidelines of the Qingdao University Affiliated Hospital in Qingdao, China, all the procedures were performed. In this study, the human B cell non-Hodgkin lymphoma cell lines SU-DHL-10 and SU-DHL4 for Type Culture Collection from the China Centre were applied. Both lymphoma cell lines were cultured in culture medium (DMEM/F12, 10\% foetal bovine serum (FBS)) under the condition of a humidified $5 \%$ $\mathrm{CO}_{2}$ chamber at $37^{\circ} \mathrm{C}$.

\section{Exosome isolation}


Exosomes were obtained from epidural ADSC supernatants by differential centrifugation. The medium was discarded when ADSCs reached $70 \%$ confluence. Then, in serum-free DMEM/F12, the cells were cultured for another $24 \mathrm{~h}$. The supernatants were collected and then cleared by sequential centrifugation

at $3,000 \mathrm{xg}$ for $30 \mathrm{~min}$ or $15,000 \mathrm{xg}$ for $30 \mathrm{~min}$. After filtration through 0.22-mm filters (Millipore, Billerica, $M A)$, the supernatants were ultracentrifuged at $120,000 \times \mathrm{g}$ for $2 \mathrm{~h}$. With sterile PBS, the exosomes were washed and collected several times. Exosome concentrations were determined with a Pierce BCA protein assay kit (Thermo Fisher Scientific).

\section{Electron microscopy}

Nearly $50 \mu \mathrm{l}$ of prepared exosomes were adsorbed and put onto formvar carbon-coated 300-mesh copper grids in approximately $10 \mathrm{~min}$. Then, at room temperature for $30 \mathrm{~min}$, the adsorbed exosomes were dried and with $3 \%$ phosphotungstic acid, they were negatively dyed. Later, the exosomes were studied through taking advantage of a transmission electron microscope (Olympus Software Imaging Solutions) at 120.0 $\mathrm{kV}$. Moreover, to capture images of the exosomes, a digital camera was used.

\section{Immunohistochemistry}

Tumour tissue samples were split into 4- $\mu \mathrm{m}$ sections and embedded in paraffin. With $100 \mu \mathrm{l}$ of a solution, antigen retrieval was conducted at $4^{\circ} \mathrm{C}$ including antibodies against Ki67 and S100A4 (1:200 dilution, Abcam MA, USA) after dehydrating the tissue samples in a graded alcohol series. Moreover, with the sections for $20 \mathrm{~min}$ at $37^{\circ} \mathrm{C}$, a diluted biotinylated secondary antibody was incubated. To visualize target proteins, haematoxylin was used as a tissue counterstain, and a fresh 3,3-diaminobenzidine (DAB) solution was also used. Two observers assessed the expression of target proteins independently by taking advantage of an Olympus FV500 optical microscope (Olympus, Tokyo, Japan) . In addition, to investigate the intensity and area of staining in five random regions (200x magnification) and evaluate the protein expression level, Image-Pro Plus 5.1was applied.

\section{Immunofluorescence}

For $24 \mathrm{~h}, \mathrm{~B}$ cell non-Hodgkin lymphoma cells were cultured with or without purified ADSC exosomes (10 $\mu \mathrm{g} / \mathrm{ml}$ ). Then, all the cells were gathered and separated, and later for $60 \mathrm{~min}$, they were fixed with $4 \%$ paraformaldehyde. The fixed cells were cut into $4-\mu \mathrm{m}$ sections and embedded in paraffin. Later, in PBS they were washed for three times and blocked with $10 \%$ goat serum for $1 \mathrm{~h}$. After that in $0.2 \%$ Triton X100 the sections were washed twice. Next, they were incubated with primary antibodies (anti-S100A4 and anti-vimentin were purchased from Abcam, MA, USA), secondary antibodies (Invitrogen) and DAPI (Guangzhou RiboBio, Guangzhou, China). Using a fluorescence microscope, images were captured.

\section{Proliferation and invasion assays}

For the following tests, B cell non-Hodgkin lymphoma cells were applied after an incubation with purified exosomes $(10 \mu \mathrm{g} / \mathrm{ml})$ for $48 \mathrm{~h}$. In previous studies, cell invasion assays and growth assays were conducted according to the manufacturer's instructions described. 


\section{EdU/PI assay}

According to the manufacturer's instructions (Guangzhou RiboBio, Guangzhou, China), 5-Ethynyl-2'deoxyuridine (EdU)/propidium iodide ( $\mathrm{PI}$ ) assays were conducted by taking advantage of a Cell-Light EdU in vitro klow cytometry kit. In brief, in a 6-well plate cells were cultured overnight, with a 20-min incubation with EdU followed. Then, they were fixed with $70 \%$ ethanol at $-20^{\circ} \mathrm{C}$ overnight and washed twice with PBS. Later, the cells were stained with a FITC-conjugated secondary antibody for $1 \mathrm{~h}$ at room temperature and denatured in $2 \mathrm{~N} \mathrm{HCl}$ for $45 \mathrm{~min}$. Moreover, with $40 \mathrm{~g} / \mathrm{ml} \mathrm{RNase} \mathrm{A}$ and $200 \mathrm{~g} / \mathrm{ml} \mathrm{PI}$, the cells were incubated for 30 min and finally analysed by flow cytometry.

\section{RNA-seq and GSEA}

SU-DHL-10 cells treated with or without ADSC exosomes were stored at $-80^{\circ} \mathrm{C}$ after being collected in $1 \mathrm{ml}$ of TRIzol reagent (Thermo Fisher Scientific). According to the instructions of the Illumina TruSeq RNA Sample Prep Kit, libraries were prepared. And on a MiSeq instrument, sequencing was conducted. At Annoroad Gene Technology Co., Ltd. (Beijing, China), the experiments were conducted. And with RSEM software, the data were analysed. All RNA-seq data can be obtained in the Sequence Read Archive (SRA) under accession nos. PRJNA65327.

Through putting the fold change data from differential expression analysis into GSEA software (Broad Institute) by taking advantage of the gene set database c2.cp.v5.1.symbols, GSEA of the RNA-seq data was conducted.

\section{shRNA transfection}

For $24 \mathrm{~h}, \mathrm{~B}$ cell non-Hodgkin lymphoma cells $\left(5 \times 10^{5}\right)$ were seeded and cultured in 10 -cm plates. Then, through making use of Lipofectamine 2000 (Invitrogen, Carlsbad, CA), they were transfected with a scrambled shRNA control (GeneChem, Shanghai, China) or S100A4-specific shRNA.

\section{qRT-PCR assay and RNA isolation}

B cell non-Hodgkin lymphoma cells were gathered. Additionally, total RNA was isolated through taking advantage of TRIzol reagent (Invitrogen, Carlsbad, USA). Later, $2 \mu \mathrm{g}$ of RNA was applied for first-strand cDNA synthesis. By using a reverse transcription kit (Toyobo, Osaka, Japan), first-strand cDNA synthesis was conducted. According to the instructions of manufacturer, quantitative real-time PCR was conducted with gene-specific TaqMan probes (Applied Biosystems) and master mix (Thermo Fisher Scientific). Gene expression was normalized to GAPDH expression. The used TaqMan probes included S100A4, Hs00656410_ce; and GAPDH, Hs02786624_g1.

\section{Western blotting}

To remove cell debris, B cell non-Hodgkin lymphoma cells were centrifuged at 10,000 x g and lysed for 10 min on ice in NP40 buffer (Beyotime, Shanghai, China). SDS-PAGE was used to separate the same amout 
$(30 \mu \mathrm{g})$ of cell extracts. And then they were transferred to polyvinylidene fluoride (PVDF) membranes (BioRad, CA, Hercules). Then, primary antibodies against $\beta$-actin (1:2,000 dilution; Abcam, MA, USA) and human S100A4, TSG101, CD63, E-cadherin and vimentin (1:1,000; Cell Signaling Technology) cultivates the PVDF membranes. Then, with the membranes, peroxidase-conjugated AffiniPure secondary IgG antibodies $(\mathrm{H}+\mathrm{L})(1: 2,000$; R\&D Systems) were incubated. On a chemiluminescence detection system, the protein-antibody complexes were detected and quantitated by using Image Lab $^{\text {TM }}$ version 5.1 software (Bio-Rad, Hercules, CA).

\section{In vivo metastasis assays and tumour growth}

The Animal Ethics Committee of the Affiliated Hospital of Qingdao University approved all experimental protocols involving animals. And from the Shanghai Animal Centre (Shanghai, China), four-week-old female BALB/c nude mice were purchased. Moreover, S100A4 shRNA/shNC SU-DHL-10 cells $\left(5 \times 10^{7}\right.$ cells/mouse) were injected subcutaneously to establish a xenograft model. After SU-DHL-10 cell injection, ADSC exosomes ( $30 \mu \mathrm{g}$, twice per week) were injected into the tumours twice per week. Six mice were included in each of the four study groups. The mice were injected with SU-DHL-10 cells ${ }^{\text {shNC }}+$ exosomes, SU-DHL-10 cells shNC + PBS, SU-DHL-10 cells ${ }^{\text {shRNA }}+$ exosomes or SU-DHL-10 cells ${ }^{\text {shRNA }}+$ PBS. After injecting cells for 7 weeks, the animals were sacrificed.

As described before, the luminescence of SU-DHL-10 cells was evaluated ${ }^{14}$.

\section{Statistical analysis}

In our study, the data are expressed as the mean \pm standard deviation. We also calculated the means after conducting no less than three independent experiments. , The significance of differences was analysed with a two-tailed Student's t-test or one-way analysis of variance, and $\mathrm{P}<0.05$ was regarded as significant.

\section{Results}

\section{ADSC exosomes enhance B cell non-Hodgkin lymphomacell invasion and proliferationin vitro}

Exosomes were purified from ADSC supernatants. To measure the diameters of the purified exosomes, an electron microscope (EM) was used. With diameters of approximately 50-150 nm, all kinds of exosomes appeared to be round vesicles (Fig. S1A). Then, western blot analysis was used to detect exosomeassociated proteins, including CD63 and TSG101 (Fig. S1B). Representative images of exosomes derived from epidural ADSCs and internalized by the B cell lymphoma cell line SU-DHL-10 were acquired at $4 \mathrm{~h}$. To research the influences of ADSC exosomes on lymphoma cell proliferation, we determined cell viability after adding $10 \mu \mathrm{g} / \mathrm{ml}$ exosomes to two lymphoma cell lines for $48 \mathrm{~h}$. We found that treatment with exosomes derived from epidural ADSCs significantly promoted proliferation in the two lymphoma cell lines (Fig. 1A). The same as this result, an EdU/PI assay showed that compared with no treatment, epidural ADSC exosome treatment raised the amount of lymphoma cells that incorporated EdU (Fig. 1B), 
indicating that exosomes increased DNA synthesis. Later, we examined whether exosomes collected from ADSC supernatants were able to augment lymphoma cell invasion. ADSC exosomes significantly increased the invasive capabilities of lymphoma cells, as shown by Transwell Matrigel invasion assays (Fig. 1C). These results revealed that epidural ADSC exosomes had a great influence on the lymphomapromoting effect of ADSCs.

\section{Global transcriptomic characterization of lymphoma cells regulated by epidural ADSC exosomes}

To better understand the changes in lymphoma cells that occurred after ADSC exosome treatment, we compared the transcriptomes of SU-DHL-10 cells cultured for $48 \mathrm{~h}$ with or without ADSC exosomes. Through RNA-seq, we found 654 upregulated and 967 downregulated genes that had changes in expression of more than two-fold (Fig. 2A). Moreover, GSEA was used to compare changed signalling pathways between lymphoma cells treated with ADSC exosomes and control SU-DHL-10 cells. The results revealed that cytokine signalling pathways associated with EMT and inflammatory responses showed the most significant upregulation in the lymphoma cells treated with ADSC exosomes (Fig. 2B). Then, we examined the top 10 elevated genes, and S100A4 was found to be related to B cell non-Hodgkin lymphoma patient survival in GEPIA (Fig. 2C). Additionally, we used an available online resource, STRING ${ }^{15}$, to visualize the protein interaction networks of S100A4 and found that S100A4 has close relationships with EMT and the cell cycle (Fig. 2D). In summary, our analyses predicted that ADSC exosomes upregulated proliferation, EMT and invasion in lymphoma cells, while S100A4 expression was upregulated significantly in this process.

\section{ADSCexosomes induceS100A4 upregulation in lymphoma cells, which is accompanied by increased EMT and MMP2/9 expression}

To have a clear idea of the function of S100A4 in epidural ADSC exosomes' lymphoma-promoting effect, S100A4, E-cadherin and vimentin expression in exosome-treated lymphoma cells was firstly examined through western blotting. Lymphoma cells which were cultured alone played the role of controls, and ADSC exosome-treated lymphoma cells expressed higher levels of S100A4 and vimentin and a lower level of E-cadherin than the control cells (Fig. 2E). Furthermore, S100A4 mRNA expression was detected in ADSC exosome-treated lymphoma cells through qRT-PCR (Fig. 2F), which revealed that ADSC exosome treatment markedly increased S100A4 mRNA expression in lymphoma cells.

\section{ADSC exosomes promote lymphoma cell proliferation, EMT and invasion viaS100A4}

To assess whether S100A4 inhibition leads to decreased proliferation, EMT and invasion in lymphoma cells, we transfected lymphoma cells with an S100A4-specific shRNA. qRT-PCR and western blot results confirmed that shRNA transfection slackened the S100A4's protein and mRNA expression in lymphoma cells (Fig. 3A-B). After confirming the inhibitory efficiency of the S100A4-specific shRNA in SU-DHL-4 and SU-DHL-10 cells, our team tested the impacts of S100A4 inhibition on the invasion and proliferation of the two lymphoma cell lines. Similar to the increased vimentin expression results, shRNA transfection decreased E-cadherin and matrix metalloproteinase (MMP) 2/9 protein expression in the two lymphoma 
cell lines (Fig. 3A, C). Then, we observed that S100A4 inhibition attenuated the significant increases in lymphoma cell proliferation and invasion in the two lymphoma cell lines caused by ADSC exosomes (Fig. $4 A-C)$. All the results suggest that S100A4 may mediate the lymphoma-promoting effects of epidural ADSC exosomes.

\section{ADSC exosomes trigger lymphoma progression by activating S100A4 in nude mouse models}

In nude mice with human SU-DHL-10 lymphoma cells, subcutaneous tumour models of lymphoma were constructed to determine how the lymphoma-promoting effects of ADSC exosomes were influenced by S100A4. With an IVIS imaging system, luciferase activity was examined to monitor tumour volume. Compared with the control group, the shRNA-transfected group exhibited slower signal increases, while the ADSC exosome-injected group displayed faster signal increases (Fig. 5A-B). Moreover, more Ki67 was found in the ADSC exosome-injected group's tumour tissues, which was accompanied by higher S100A4, lower E-cadherin expression, and vimentin and MMP2/9 expression. The results suggested that S100A4 inhibition significantly reduced the growth, EMT and invasion of lymphoma (Fig. 5C). Thus, ADSC exosomes promoted lymphoma metastasis and growth. In addition, these effects were counteracted by S100A4 inhibition.

\section{Discussion}

In the current study, we used RNA-seq to explore the mechanisms underlying the malignancy-promoting effects of epidural ADSC exosomes on B cell lymphomas. According to the RNA-seq results for lymphoma cells treated with ADSC exosomes, GSEA revealed that the activation of EMT-associated pathways in exosome-treated lymphoma cells was accompanied by increased S100A4 expression. Enhanced EMT plays important roles in the metastasis of aggressive B cell lymphomas, which may explain the special spinal metastatic affinity. Our data link S100A4 upregulation to the metastasispromoting effect of epidural ADSC exosomes, further implicating S100A4 as an important therapeutic target. Our findings support the concept that the microenvironment in parts of cancer cells enhanced cancer cell metastasis and maintained cancer cell growth and survival through secretion, particularly exosome secretion.

In specific organs, a premetastatic niche is a microenvironment prepared for colonizing circulating tumour cells ${ }^{16}$. Our previous studies showed that conditioned medium from epidural ADSCs promoted primary lung cancer forming premetastatic niches in the spine ${ }^{6}$. Increasing evidence suggests that premetastatic niche formation may determine metastatic organotropism in cancer patients ${ }^{17}$. In addition, several studies have revealed that ADSCs from subcutaneous adipose tissue promote the growth of acute lymphoblastic leukaemia cells and $T$ cell lymphoma cells ${ }^{18,19}$. It is still unknown that whether $B$ cell lymphoma which is the most common type non-Hodgkin's lymphoma, has a premetastatic niche in the spine that involves epidural ADSCs. Exosomes are extracellular vesicles with various biological functions. Moreover, exosomes have great influences on premetastatic niches, determine organotropic metastasis and cause extracellular matrix remodelling ${ }^{20,21}$. In this research, we isolated exosomes from ADSCs, and 
lymphoma cell EMT was induced by these exosomes. Although this finding still requires further investigation, RNA-seq results indicated that elevated S100A4 expression in lymphoma cells might be the cause of enhanced EMT, which was accompanied by the downstream activation of MMP2/9.

Metastasis is directly associated with cancer progression and is the main cause of cancer-related death. Moreover, EMT enables cells to migrate and invade in cancer ${ }^{22}$. EMT results in the upregulation of mesenchymal markers' expression, containing vimentin, and downregulation of key adherence proteins' expression, containing E-cadherin, which enhances the invasive and migratory properties of tumour cells $^{12,23}$. S100 is a family of low-molecular weight proteins consisting of 25 closely related members in humans ${ }^{24}$. It has been revealed that $\mathrm{S} 100$ proteins are related to EMT regulation and numerous biological processes that possibly contain cell survival, cell cycle regulation, apoptosis and cell growth and motility ${ }^{25,26}$. S100A4 is a significant member in the $\$ 100$ family, and it plays a role in increasing tumour metastasis and progression ${ }^{9}$. In the early prediction of cancer metastasis and diagnosis of cancer, S100A4 has become a promising candidate biomarker; thus, S100A4 is likely to be a therapeutic target ${ }^{27}$.

Altogether, in this study, we focused on the special metastatic pattern of non-Hodgkin lymphoma in the vertebral body, and epidural ADSCs were found to be involved in the formation of the premetastatic niche in B cell non-Hodgkin lymphoma. Our results support the conclusion that exosomes secreted by ADSCs can activate the expression of S100A4 in lymphoma cells, which fosters lymphoma metastasis and growth. Furthermore, ADSC exosomes have been displayed so as to induce EMT and the expression of growth factors, including MMPs and vimentin, in tumour cells, which could partly explain the increased proliferation and invasion observed. However, our knowledge on S100A4's exact mechanism that influences the organotropic metastasis of lymphoma is limited. In the tumour microenvironment, our findings could result in the design of lymphoma therapies targeting protein modifications to change ADSCs' tumour-promoting effects.

\section{Declarations}

\section{Ethics approval and consent to participate}

The study was approved by the Ethics Committee of the Qingdao University Affiliated Hospital. All participants provided a written informed consent.

\section{Consent for publication}

Not applicable.

\section{Availability of data and materials}

The datasets used and/or analyzed during the current study are available from the corresponding author on reasonable request. 


\section{Competing interests}

The authors declare no potential conflicts of interest.

\section{Funding}

This study was supported by the National Natural Science Foundation of China $(81802190,81772412$, $81871804,81672200)$. All funders had no role in the design of the study and collection, analysis, and interpretation of data and in writing the manuscript.

\section{Authors' contributions}

Conceived and designed the experiments: BC and XM. Performed the experiments: YW, YC, HX and ZG. Collected samples: XR, YX, CZ, KZ and GZ. Analyzed the data: YC. Wrote the paper: YW and YC. All authors read and approved the final manuscript.

\section{Acknowledgements}

The present study was supported by the national key clinical speciality construction project of China. We thank the Department of Orthopaedic Surgery, the Affiliated Hospital of Qingdao University, Qingdao, China, for providing tissue samples.

\section{References}

1. Cole, J.S. \& Patchell, R.A. Metastatic epidural spinal cord compression. The Lancet Neurology7, 459466 (2008).

2. B, A.J.L.B.M.B.H.M. Spinal cord compression in patients with advanced metastatic cancer: "all I care about is walking and living my life". JAMA299, 937-946 (2008).

3. Perrin, R.G. \& Laxton, A.W. Metastatic spine disease: epidemiology, pathophysiology, and evaluation of patients. Neurosurg Clin N Am15, 365-373 (2004).

4. Dropcho, E.J. Neurologic complications of lung cancer. Handb Clin Neuro/119, 335-361 (2014).

5. Koeller, K.K. \& Shih, R.Y. Extranodal Lymphoma of the Central Nervous System and Spine. Radiol Clin North Am54, 649-671 (2016).

6. Wang, Y., et al. Epidural adipose tissue-derived mesenchymal stem cell activation induced by lung cancer cells promotes malignancy and EMT of lung cancer. Stem cell research \& therapy10, 168 (2019).

7. Li, I. \& Nabet, B.Y. Exosomes in the tumor microenvironment as mediators of cancer therapy resistance. Mol Cancer18, 32 (2019).

8. Guo, Y., et al. Effects of exosomes on pre-metastatic niche formation in tumors. Mol Cancer18, 39 (2019). 
9. Li, F., et al. S100A4-MYH9 Axis Promote Migration and Invasion of Gastric Cancer Cells by Inducing TGF-beta-Mediated Epithelial-Mesenchymal Transition. Journal of Cancer9, 3839-3849 (2018).

10. Heizmann, C.W. Ca(2+)-Binding Proteins of the EF-Hand Superfamily: Diagnostic and Prognostic Biomarkers and Novel Therapeutic Targets. Methods in molecular biology1929, 157-186 (2019).

11. Tamaki, Y., et al. Metastasis-associated protein, S100A4 mediates cardiac fibrosis potentially through the modulation of p53 in cardiac fibroblasts. J Mol Cell Cardio/57, 72-81 (2013).

12. Piera-Velazquez, S. \& Jimenez, S.A. Endothelial to Mesenchymal Transition: Role in Physiology and in the Pathogenesis of Human Diseases. Physiol Rev99, 1281-1324 (2019).

13. Fei, F.Q., J.; Zhang, M.; Li, Y.; Zhang, S. S100A4 in cancer progression and metastasis: A systematic review. oncotarget8, 73219-73239. (2017).

14. Tang, H., Chu, Y., Huang, Z., Cai, J. \& Wang, Z. The metastatic phenotype shift towards myofibroblast of adipose-derived mesenchymal stem cells promotes ovarian cancer progression. Carcinogenesis (2019).

15. Szklarczyk, D., et al. STRING v11: protein-protein association networks with increased coverage, supporting functional discovery in genome-wide experimental datasets. Nucleic Acids Res47, D607D613 (2019).

16. Liu, Y. \& Cao, X. Characteristics and Significance of the Pre-metastatic Niche. Cancer cel/30, 668-681 (2016).

17. Chen, W., Hoffmann, A.D., Liu, H. \& Liu, X. Organotropism: new insights into molecular mechanisms of breast cancer metastasis. npj Precision Oncology2(2018).

18. Lee, M.W., et al. Human Adipose Tissue Stem Cells Promote the Growth of Acute Lymphoblastic Leukemia Cells in NOD/SCID Mice. Stem Cell Rev Rep14, 451-460 (2018).

19. Xu, L., et al. Hypoxia-induced secretion of IL-10 from adipose-derived mesenchymal stem cell promotes growth and cancer stem cell properties of Burkitt lymphoma. Tumour biology : the journal of the International Society for Oncodevelopmental Biology and Medicine37, 7835-7842 (2016).

20. Tominaga, N., et al. Brain metastatic cancer cells release microRNA-181c-containing extracellular vesicles capable of destructing blood-brain barrier. Nature communications6, 6716 (2015).

21. Syn, N., Wang, L., Sethi, G., Thiery, J.P. \& Goh, B.C. Exosome-Mediated Metastasis: From EpithelialMesenchymal Transition to Escape from Immunosurveillance. Trends in pharmacological sciences37, 606-617 (2016).

22. Pastushenko, I., et al. Identification of the tumour transition states occurring during EMT. Nature556, 463-468 (2018).

23. Wang, Y., Tan, J., Wu, H. \& Yi, C. High Glucose Promotes Epithelial-Mesenchymal Transition, Migration and Invasion in A20 Murine Di ff use Large B-Cell Lymphoma Cells Through Increased Expression of High Mobility Group AT-Hook 2 (HMGA2). Med Sci Monit25, 3860-3868 (2019).

24. Heizmann, C.W. S100 proteins: Diagnostic and prognostic biomarkers in laboratory medicine. Biochim Biophys Acta Mol Cell Res1866, 1197-1206 (2019). 
25. Raffat, M.A., et al. S100 proteins in oral squamous cell carcinoma. Clin Chim Acta480, 143-149 (2018).

26. Verma, R., Verma, P., Budhwar, S. \& Singh, K. S100 proteins: An emerging cynosure in pregnancy \& adverse reproductive outcome. Indian J Med Res148, S100-S106 (2018).

27. $\mathrm{Xu}, \mathrm{H}$., et al. S100A4 participates in epithelial-mesenchymal transition in breast cancer via targeting MMP2. Tumour biology : the journal of the International Society for Oncodevelopmental Biology and Medicine37, 2925-2932 (2016).

\section{Figures}

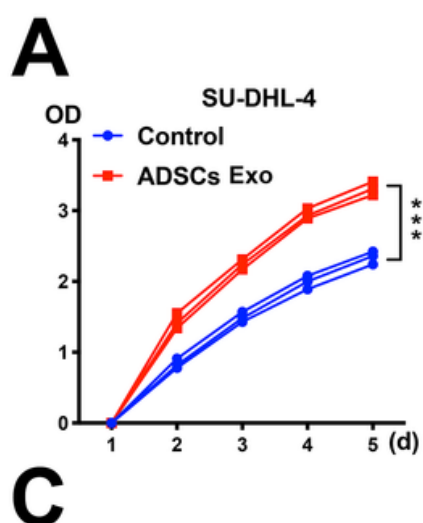

Control

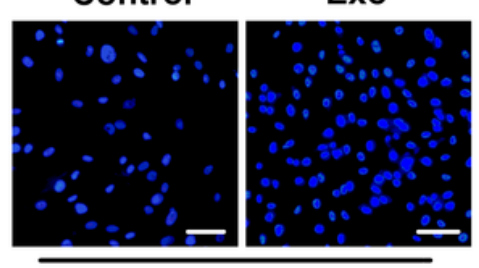

SU-DHL-4
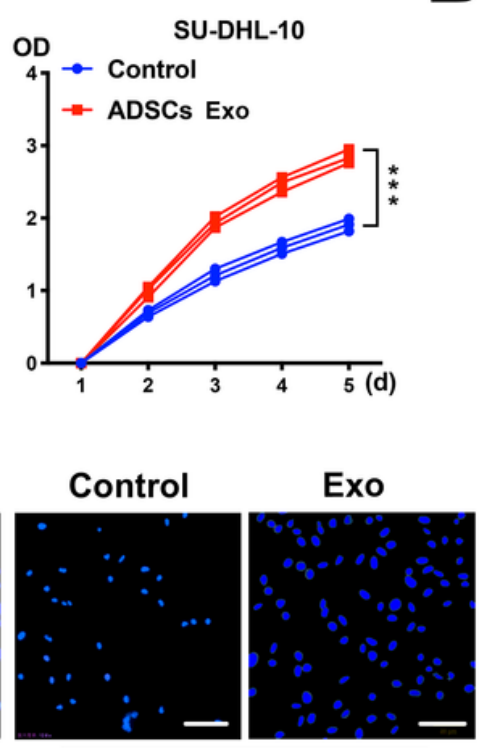

SU-DHL-10
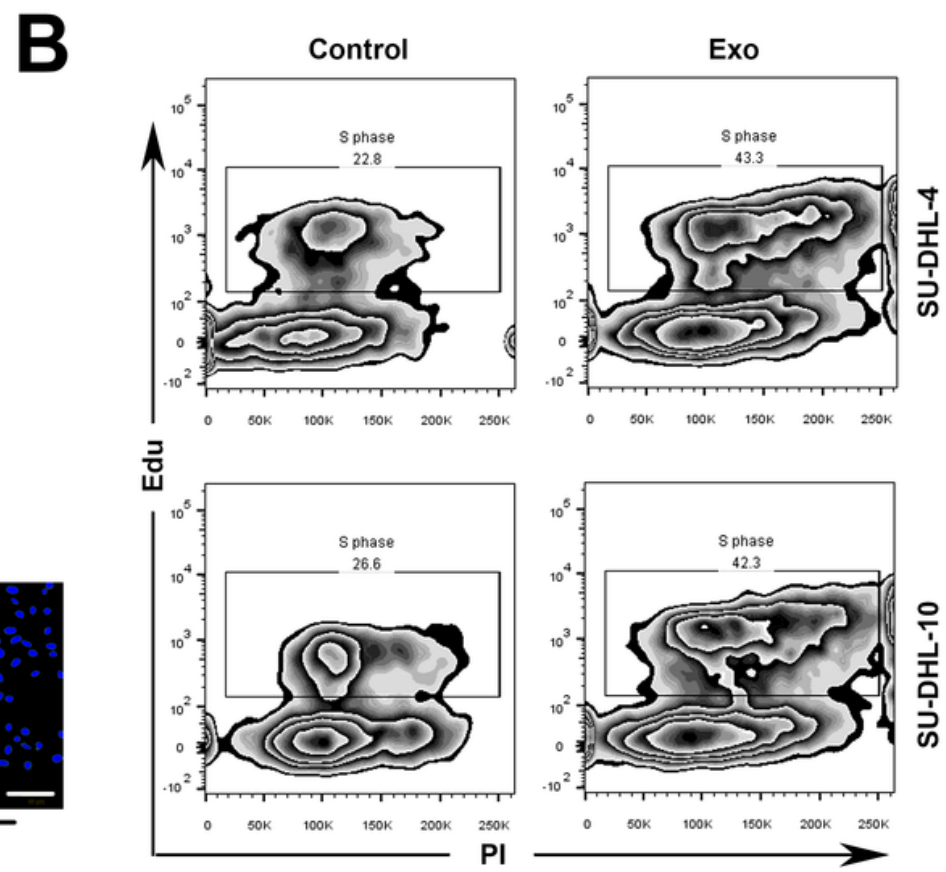

\section{Figure 1}

ADSC exosomes promote the proliferation and invasion of B cell lymphoma cells. (A): ADSC exosomes's influences on lymphoma cell proliferation were assessed by using a CCK8 assay. Lymphoma cells were treated with or without ADSC exosomes, and the densities of the two groups at $450 \mathrm{~nm}$ were studied. Data collected from the three respective experiments are shown. (B): EdU flow cytometry analysis on the EdU positive lymphoma cells treated with or without ADSC exosomes. (C): Comparison of lymphoma cells' invasion treated with or without ADSC exosomes using Transwell compartments. ${ }^{\star} * \star \quad P<0.001$. 


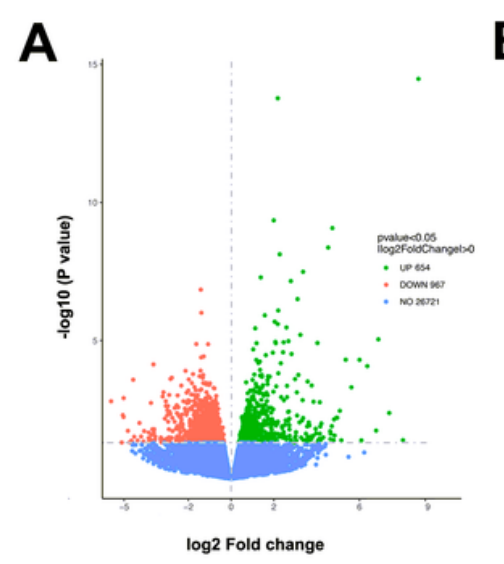

B

Pathways upregulated in ADSC Exosomes treated SU-DHL-10 cells
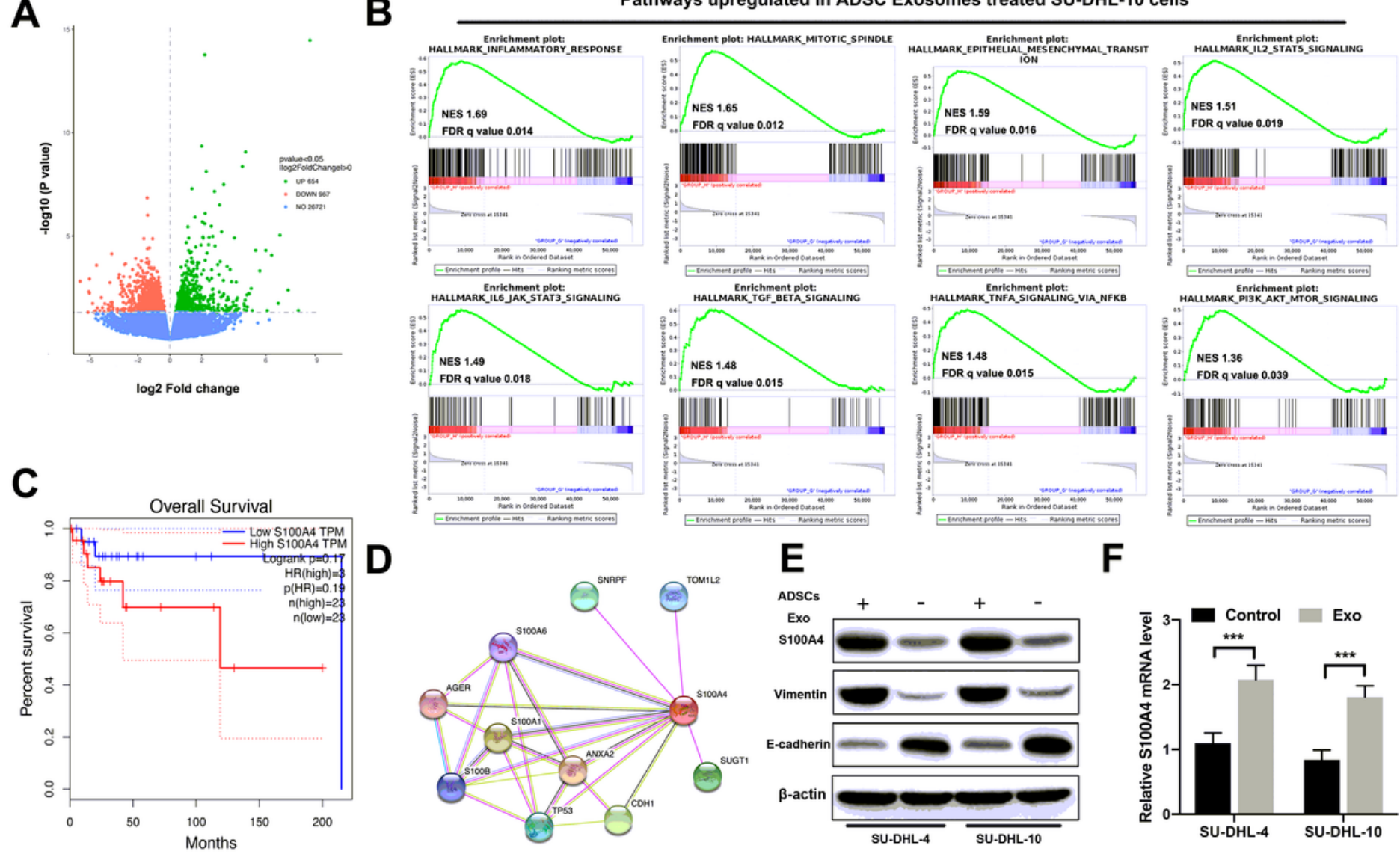

$\mathbf{F}$

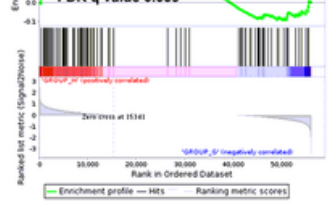

\section{Figure 2}

Global transcriptome characterization of lymphoma cells regulated by ADSC exosomes and upregulation of S100A4 expression by ADSC exosomes. (A): The volcano map of differentially expressed genes under RNA seq in SU-DHL-10 cells treated with or without ADSC exosomes. (B): GSEA of most up-regulated pathways in SU-DHL-10 cells treated with ADSC exosomes compared with SU-DHL-10 cells cultured alone. (C): S100A4 was examined to related to B cell non-hodgkin lymphoma survival in GEPIA website. (D): S100A4 protein interaction networks were examined in STRING. (E): Significant increases of S100A4 and Vimentin, and decrease of E-cadherin expression were found in two lines lymphoma cells treated with ADSC Exo under hypoxic condition by western blotting. $(F)$ : Real-time PCR displays the relative mRNA expression of S100A4 in lymphoma cells treated with or without ADSC exosomes. The results are expressed as the means $\pm S D$. ${ }^{* \star *} P<0.001$. 

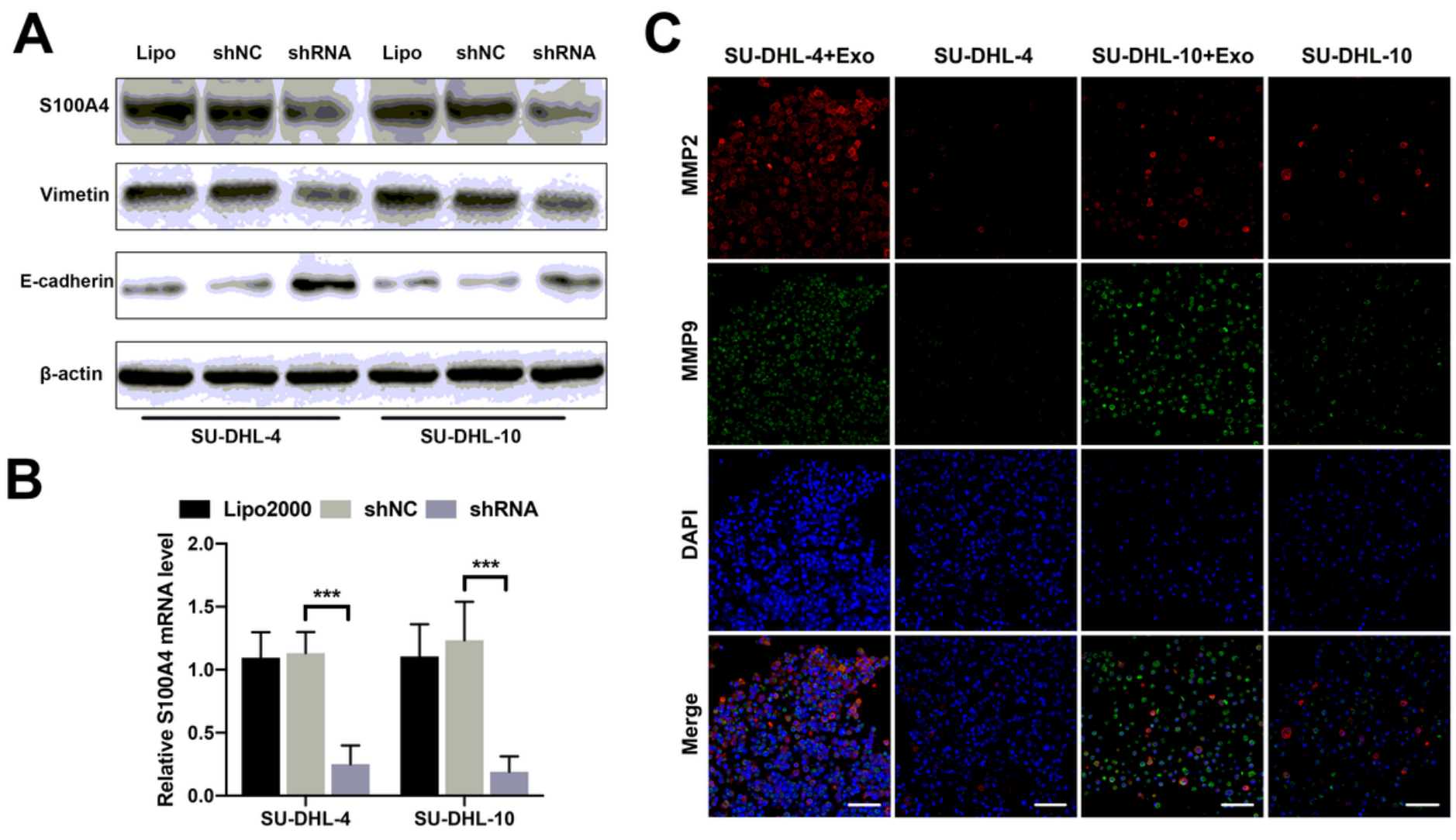

\section{Figure 3}

ADSC exosomes induce S100A4 upregulation in lymphoma cells, accompanied by increased vimentin and MMP2/9 expression. (A): Western blotting confirmed that in lymphoma cells, S100A4 shRNA treatment downregulated S100A4, vimentin expression and upregulated E-cadherin expression. (B): Realtime PCR displays the relative mRNA expression of S100A4 in lymphoma cells treated with S100A4 shRNA. (C): Representative IF images of MMP2 and MMP9 in lymphoma cells treated with S100A4 shRNA. Scale bar $=25 \mu \mathrm{m}$. *** $\mathrm{P}<0.001$. 
A
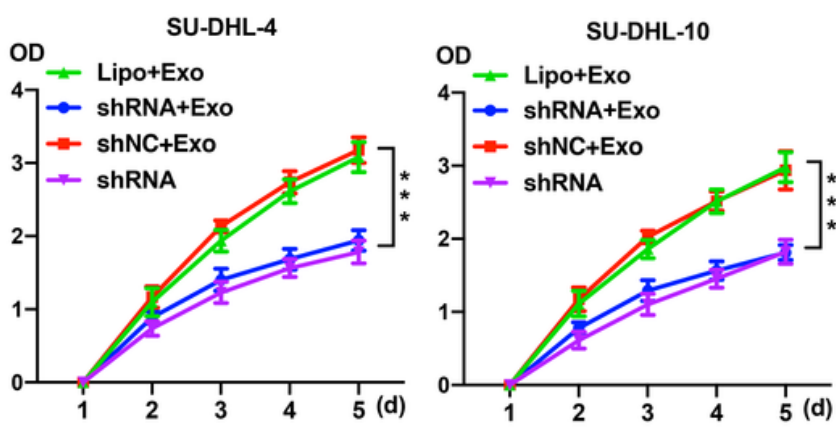

C

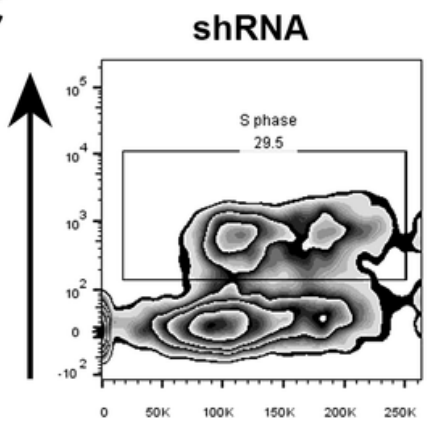

궁

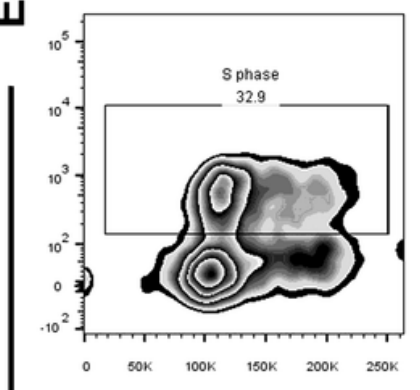

B
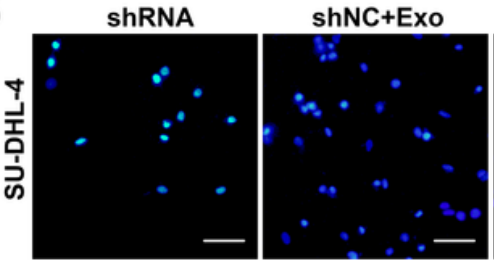

Lipo+Exo

ShRNA+Exo
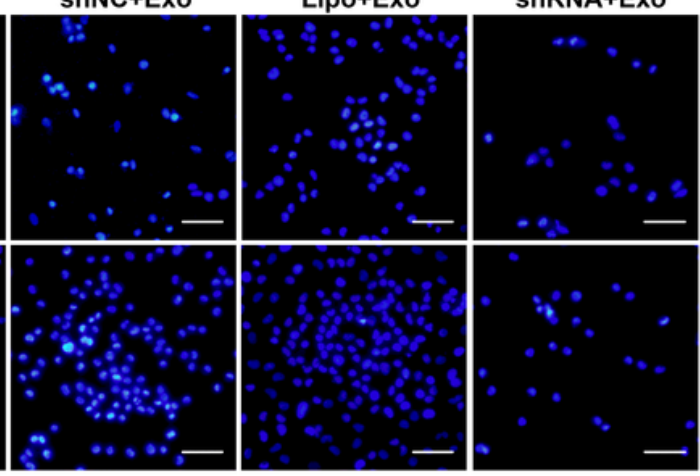

Lipo+Exo
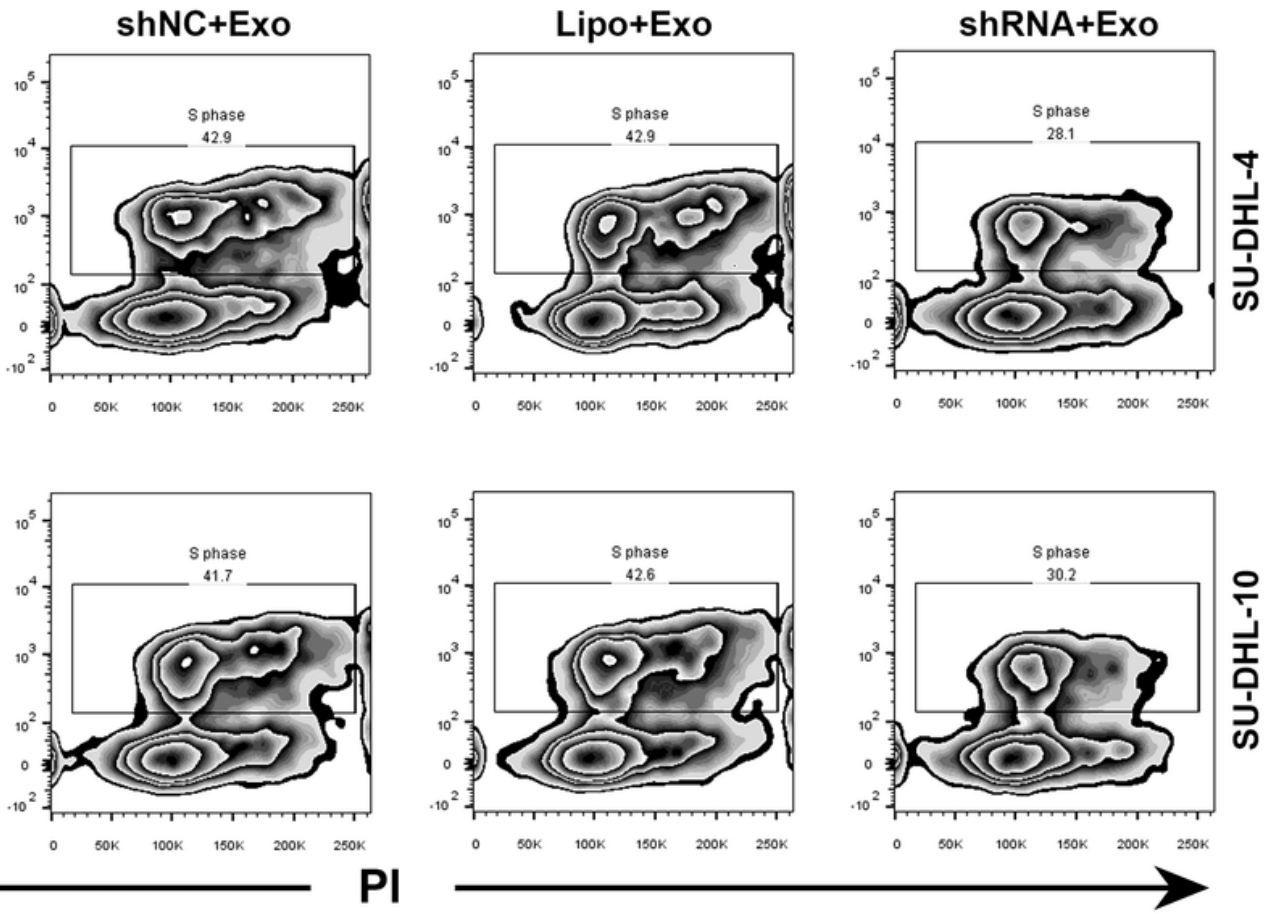

\section{Figure 4}

S100A4 inhibition by shRNA decrease the proliferation and invasion of lymphoma cells. (A): S100A4 inhibition's effects on SU-DHL-4 and SU-DHL-10 cell proliferation were assessed through a CCK8 assay. Data collected from the three respective experiments are reflected. (B): Representative images of invaded cells after S100A4 knockdown in the Transwell invasion assay. (C): Representative images of EdU flow cytometry analysis on the S100A4 knockdown lymphoma cells. ${ }^{\star * \star} \mathrm{P}<0.001$. 

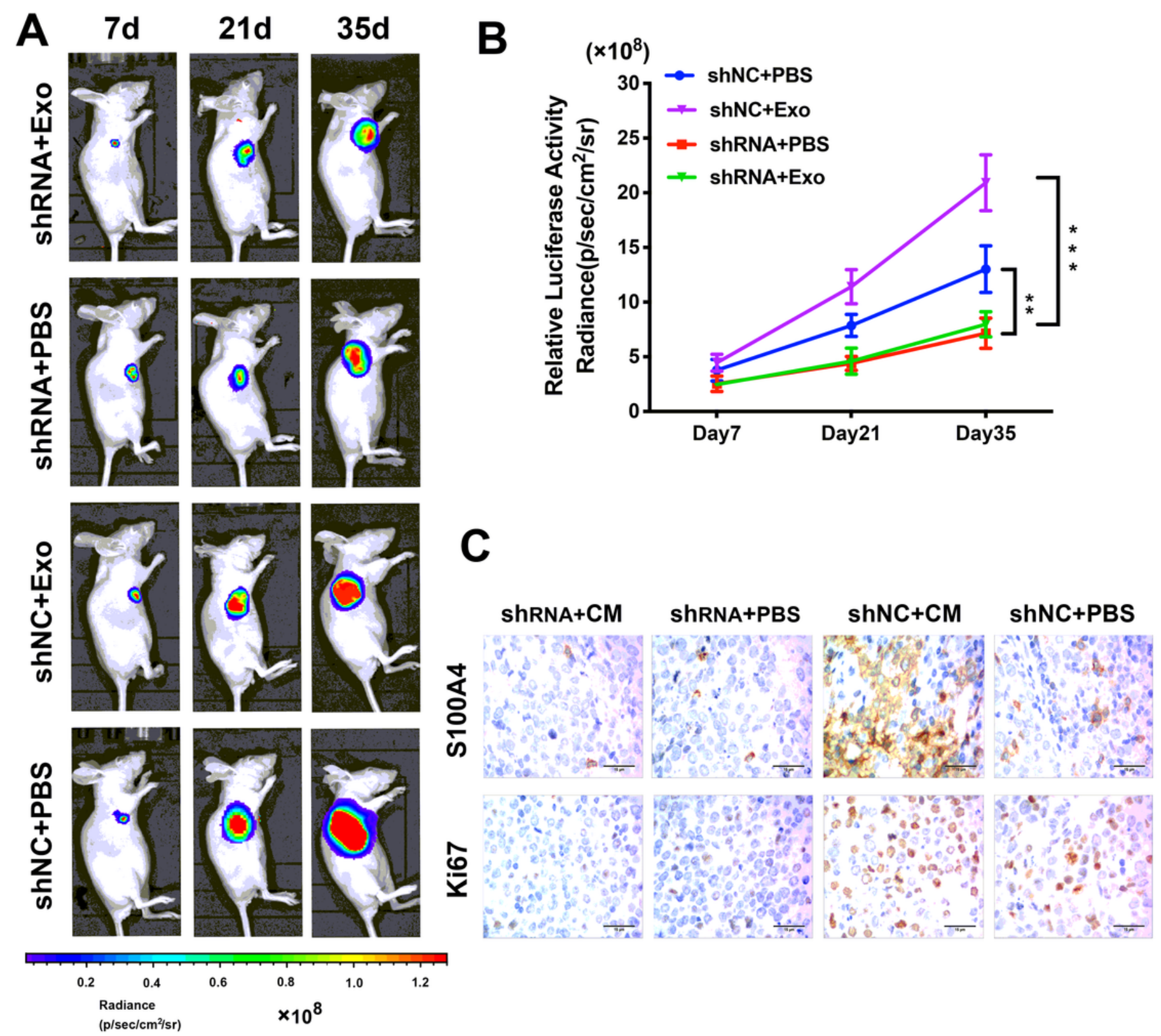

Figure 5

S100A4 mediates the promotion effects of ADSC exosomes on lymphoma growth in subcutaneous models of nude mice. (A): To inspect lymphoma xenograft luminescence activity, an in vivo imaging system was applied. The result indicated tumour growth. (B): To examine the tumour bioluminescence intensity every two weeks, living Image Software was applied. Besides, the quantitation of the normalized image counts is displayed. (C): In subcutaneous lymphoma xenografts, immunohistochemical analysis on S100A4 and Ki67 expression is reflected. Scale bar: $25 \mu \mathrm{m}$. ${ }^{\star *} \mathrm{P}<0.01$.

\section{Supplementary Files}

This is a list of supplementary files associated with this preprint. Click to download. 
- Fig.S1.tif

Page 17/17 